\title{
Impact of Pro Bono Clinic on Attitudes, Beliefs, and Confidence Towards Cultural Competence in First-year Doctoral Physical Therapy Students
}

\author{
Scarlett Morris \\ University of Kansas, smorris6@kumc.edu \\ RuiPing Xia \\ Franklin Pierce University, XiaR@franklinpierce.edu \\ Troy Klaassen \\ University of Saint Mary, troyklaassen10@gmail.com \\ Trey Johnson \\ University of Saint Mary, treyjohnson545@gmail.com
}

Follow this and additional works at: https://nsuworks.nova.edu/ijahsp

Part of the Other Rehabilitation and Therapy Commons, Physical Therapy Commons, and the Scholarship of Teaching and Learning Commons

\section{Recommended Citation}

Morris S, Xia R, Klaassen T, Johnson T. Impact of Pro Bono Clinic on Attitudes, Beliefs, and Confidence Towards Cultural Competence in First-year Doctoral Physical Therapy Students. The Internet Journal of Allied Health Sciences and Practice. 2021 Oct 01;19(4), Article 12.

This Manuscript is brought to you for free and open access by the College of Health Care Sciences at NSUWorks. It has been accepted for inclusion in Internet Journal of Allied Health Sciences and Practice by an authorized editor of NSUWorks. For more information, please contact nsuworks@nova.edu. 


\title{
Impact of Pro Bono Clinic on Attitudes, Beliefs, and Confidence Towards Cultural Competence in First-year Doctoral Physical Therapy Students
}

\begin{abstract}
Purpose: Cultural competence is an essential skill for healthcare providers in our increasingly ethnically diversified society. There is an unmet need for educating future culturally competent physical therapists. The purpose of this study was to investigate the impact of pro bono clinic on cultural competence in first-year doctoral physical therapy students. Method: Forty-two participants completed a survey before and after attending three individual sessions of mentored pro bono clinical experiences during their first semester. The survey assessed participants' attitudes and beliefs (Part I), and self-perceived level of confidence (Part II) towards cultural competence. Wilcoxon signed-rank tests were performed. Results: The mean scores increased from pretest $(4.13 \pm 0.55)$ to posttest $(4.22 \pm 0.48)$ for Part I (5: highest scale) and from $7.34 \pm 0.96$ to $8.01 \pm 0.79$ for Part II (10: highest level) but were not significant. Overall findings showed a positive impact which was reflected by students' narrative comments post-participation (Part III). Conclusion: This study is novel due to limited evidence in this area, especially the effect of an early intervention. Pro bono clinic participation may be an effective approach that could be incorporated early in curriculum across allied health science education.
\end{abstract}

\section{Author Bio(s)}

Scarlett Morris, PT, DPT, is a Clinical Assistant Professor at the University of Kansas Medical Center in Kansas City, KS. She is a Board-Certified Orthopaedic Specialist and holds a certificate in orthopaedic manual therapy.

RuiPing Xia, MS, PhD, is an Associate Professor in Doctor of Physical Therapy Program at Franklin Pierce University in Goodyear, AZ.

Troy Klaassen, PT, DPT is a graduate of the University of Saint Mary, Leavenworth, KS and currently works as a physical therapist and clinic director in Colorado.

Trey Johnson, PT, DPT is a graduate of the University of Saint Mary, Leavenworth, KS and currently works as a physical therapist at Great Plains Health Sports and Therapy Center in North Platte, Nebraska.

\section{Acknowledgements}

The authors would like to thank the study participants from the University of Saint Mary DPT program and acknowledge the St. Vincent Clinic, both in Leavenworth, KS. 


\title{
IIJAHSP \\ The Internet Joumnal of Allied Health Sciences and Practice \\ Dedicated to allied health professional practice and education \\ Vol. 19 No. 4 ISSN 1540-580X
}

\section{Impact of Pro Bono Clinic on Attitudes, Beliefs, and Confidence Towards Cultural Competence in First-year Doctoral Physical Therapy Students}

\begin{abstract}
Scarlett Morris ${ }^{1}$
RuiPing Xia ${ }^{2}$

Troy Klaassen ${ }^{3}$

Trey Johnson ${ }^{3}$

1. University of Kansas

2. Franklin Pierce University

3. University of Saint Mary

United States

ABSTRACT

Purpose: Cultural competence is an essential skill for healthcare providers in our increasingly ethnically diversified society. There is an unmet need for educating future culturally competent physical therapists. The purpose of this study was to investigate the impact of pro bono clinic on cultural competence in first-year doctoral physical therapy students. Method: Forty-two participants completed a survey before and after attending three individual sessions of mentored pro bono clinical experiences during their first semester. The survey assessed participants' attitudes and beliefs (Part I), and self-perceived level of confidence (Part II) towards cultural competence. Wilcoxon signed-rank tests were performed. Results: The mean scores increased from pretest $(4.13 \pm 0.55)$ to posttest (4.22 \pm 0.48 ) for Part I (5: highest scale) and from $7.34 \pm 0.96$ to $8.01 \pm 0.79$ for Part II (10: highest level) but were not significant. Overall findings showed a positive impact which was reflected by students' narrative comments post-participation (Part III). Conclusion: This study is novel due to limited evidence in this area, especially the effect of an early intervention. Pro bono clinic participation may be an effective approach that could be incorporated early in curriculum across allied health science education.
\end{abstract}

Keywords: pro bono, cultural competence, physical therapy education, clinical education 


\section{INTRODUCTION}

Population diversity is ever increasing in the United States. It is expected that by 2060, the non-Hispanic whites in the U.S. will decline from an estimated $62 \%$ to $44 \%$, and that the U.S. will become a "plurality of racial and ethnic groups," with no single group dominating the "majority" by $2044 .{ }^{1}$ The U.S. Census Bureau also projects an increased population with a foreign-born status. As the American population continues to diversify in these ways, it is pivotal that healthcare providers become culturally competent in healthcare delivery.

The National Institutes of Health $(\mathrm{NIH})$ describes culture as the "personal identification, language, thoughts, communications, actions, customs, beliefs, values, and institutions that are often specific to ethnic, racial, religious, geographic, or social groups." Culturally competent healthcare, sometimes also referred to as cultural respect or cultural humility, may be defined more broadly to include factors beyond culture, such as socioeconomic status (SES). Evidence has shown that a low SES has been associated with poorer health outcomes and biased clinical decision making. ${ }^{3}$ On the contrary, culturally competent healthcare has been linked to decreased medical errors, fewer delays in care, and increased patient safety, engagement and adherence. ${ }^{4}$ According to the $\mathrm{NIH}$, awareness of culturally competent care is "critical to reducing health disparities and improving access to high-quality health care." ${ }^{2}$ Improving cultural competence in healthcare providers as well as healthcare systems has been proposed to be an effective approach to increasing quality of care and eliminating health disparities. ${ }^{5}$ Therefore, cultural competence is increasingly recognized as a critical skill for clinical practice across all health professions including physical therapy.

The American Physical Therapy Association (APTA) describes cultural competence as "an ongoing process, not an end point" and a "necessary skill to ensure best practice."6 They also provide general guidance for teaching and improving cultural competence on their website. ${ }^{7}$ There are several standards and elements associated with cultural competence required by the Commission on Accreditation in Physical Therapy Education (CAPTE), the agency that grants accreditation status to education programs for physical therapists and physical therapist assistants. ${ }^{8}$ CAPTE standards indirectly specify that physical therapy services should incorporate cultural differences of patients/clients and that physical therapists should provide pro bono services to meet health needs of people who are socioeconomically disadvantaged (7D4 and 7D5). The document also directly states that recruitment and admission of increased diversity (e.g., race, ethnicity, socioeconomic status, cultural and individual differences) in student body be consistent with societal needs for physical therapy services for a diverse population (Standard 5). This statement implies the importance of recognition and inclusion of diversity in a broad context and the impact of an early exposure to diversity beginning in the admissions stage.

While a teaching construct for cultural competence in physical therapy education is still developing, established models are used to teach cultural competence in other healthcare professions. ${ }^{9-13}$ Furthermore, systematic review studies reported training and development of cultural competence for health professionals in rehabilitation and in general practitioners. ${ }^{14,15}$ Individual studies included in these reviews varied largely in the design approach and the types of training. Intervention programs were primarily educational sessions such as lectures as a part of a general training program, cultural competence workshops, work-based programs, small group work, cultural school, and cultural sensitivity programing. Outcome measures mainly included cultural knowledge, attitudes, competence, and skills in healthcare providers. Overall findings of the study in rehabilitation were inconclusive due to inconsistency of the results. ${ }^{14}$ The study by Watt et al. (2016) concluded that there was a lack of focus and formal training in cross-cultural practice even though cultural competence is recognized to be of vital importance. ${ }^{15}$ It should be noted that the training programs described in these studies did not include pro bono clinic.

Many physical therapist educational programs have utilized an international immersion model by offering students an opportunity to participate in international service-learning experience with respect to providing care to populations who are underserved, impoverished, or underrepresented. ${ }^{16-19}$ Despite students' improvement in cultural competence, international service experiences are not realistically available to all students, considering the high cost and other logistical factors associated with international travel and service. Therefore, it is pivotal to explore more practical ways, such as pro bono clinic, to expose students to cultural dimensions and teach students cultural awareness, knowledge, diversity, and other pertinent elements in order for students to become culturally competent.

A commentary titled: "Is There a Best Practice Educational Model to Facilitate Students Achieving Cultural Competence?" highlighted three published articles that presented three different models: 1 . An experiential model via international cultural immersion; 2. A global model via study abroad; and 3. A culturally sensitive academic environment within the Doctor of Physical Therapy (DPT) program. The variety of approaches presented in this commentary reveals a gap in the literature: Which model, if any, is superior? And what is the necessary dosage or length of exposure needed for each type to optimize effectiveness? ${ }^{20}$ 
Other approaches involve student participation in classroom and cross-cultural service-learning activity or placement in pro bono clinic prior to extensive fulltime clinical experiences. ${ }^{21-25}$ This type of pre-clinical experience is often referred to as Integrated Clinical Experience, meaning that it is imbedded within the didactic portion of the curriculum. The general goal of these abbreviated clinical experiences is to broaden students' contextualization of skills and values learned in the classroom. Pro bono clinic involvement during the didactic education has been used to engage the topics of professionalism and cultural competence, and often provides students with an opportunity to practice clinical skills. ${ }^{23,24}$ Studies involving these curriculum-integrated pro bono clinic models showed that exposing students to pro bono clinical practice before going out on their first clinical rotation was beneficial for the development of cultural diversity, competence, and other APTA core values such as social responsibility. ${ }^{26}$

Palombaro et al. utilized various curricular activities throughout the DPT education and examined their impact on students' cultural competence at the end of the curriculum. ${ }^{27}$ The activities covered several cross-cultural programs including voluntary pro bono clinic service and pro bono clinic student leadership participation. This study found a significant increase in cultural competence based on student self-assessment, particularly in those students that took a leadership role in the pro bono clinic. Using a retrospective analysis of student clinical performance ratings, a recent study showed that required participation in a student-led pro bono clinic during the didactic education had a positive outcome on overall long-term clinical performance and cultural competence. $^{28}$

The findings of the above studies may suggest a potential benefit of the pro bono clinic experiences to student development in cultural competence and other clinical skills. Considering the confounding influences of many other cross-cultural programs and the retrospective nature of the study, there is a critical need to gather more evidence on this topic. ${ }^{27-28}$ Therefore, the purpose of this study was to investigate the impact of participation in a pro bono clinic on cultural competence early in the curricula, particularly in first-year DPT students. We hypothesized that participation in a pro bono clinic during the first semester would positively influence students' attitudes, beliefs, views of cultural background and diversity and improve their cultural competence. To our best knowledge, no study has examined the influence of the pro bono clinic alone, as part of the DPT curriculum, on students' cultural competence.

\section{METHODS}

\section{Subjects}

The sample consisted of 42 first-year DPT students. Participants were $87.8 \%$ White/Caucasian, $4.9 \%$ Black/African American, 4.9\% Hispanic/Latino, and 1.4\% Asian/Pacific Islander American and had a mean age of $24.5 \pm 2.24$ years (see Table 1). Participants were recruited from the University of Saint Mary (USM). The inclusion criterion for the study was enrollment as a firstyear DPT student at USM. The study was approved by USM's Institutional Review Board. Informed consent was integrated into the beginning of the electronic survey and obtained from all participants.

Table 1. Participants' demographic data including gender and race/ethnicity.

\begin{tabular}{|l|c|}
\hline \multicolumn{1}{|c|}{ Characteristics } & $\mathbf{N}=\mathbf{4 2}$ \\
\hline Mean $\pm(\mathrm{SD})$ age in years & $24.5 \pm 2.24$ \\
\hline Sex & $\mathbf{n ~ ( \% )}$ \\
\hline Men & $13(31.7 \%)$ \\
\hline Women & $27(65.9 \%)$ \\
\hline Not specified & $1(2.4 \%)$ \\
\hline & \\
\hline Race/Ethnicity & $\mathbf{n ~ ( \% )}$ \\
\hline White/Caucasian American & $36(87.8 \%)$ \\
\hline Black/African American & $2(4.9 \%)$ \\
\hline Hispanic/Latino & $2(4.9 \%)$ \\
\hline Asian/Pacific Islander American & $1(1.4 \%)$ \\
\hline
\end{tabular}

\section{Study Design and Procedures}

The study utilized a single-group pretest and posttest survey design in order to assess changes in cultural competence as a result of participation in the pro bono clinic during a 16-week semester. This particular study revolved around a service-learning experience offered at a local pro bono clinic in Leavenworth, Kansas, as part of a required course, in the fall semester of 2017. 
Over this period, each student was required to assist a second-year DPT student in providing care at the pro bono clinic on at least 3 occasions. The pretest survey was administered in class at the beginning of the semester prior to participation in the pro bono clinic, and the posttest survey was distributed at the end of the semester after the completion of their participation. Survey data were collected using Qualtrics (Qualtrics®, Provo, Utah).

\section{Outcome Measures}

Outcomes of the study included attitudes, beliefs, and self-reported level of confidence regarding cultural competence for both pretest and posttest. The entire survey consisted of 23 items divided into two parts. Part I consisted of 13 statements related to cultural attitudes and beliefs that were rated on a Likert scale from 1 to $5 ; 1=$ strongly disagree, $2=$ disagree, $3=$ neutral, $4=$ agree, 5 = strongly agree. Part II contained 10 questions that aimed to acquire participants' self-perceived level of confidence regarding cultural competence. This Part used an 11-point scale, with 0 being no confidence at all and 10 being full confidence. Higher rating in either Part represented higher levels of perceived cultural competence. Students' narratives on participation were collected for post-test as Part III (see Appendix for complete survey).

\section{Survey Validation}

A survey was independently constructed to test our hypothesis and achieve the objective of this study. ${ }^{29-31}$ With respect to question validation, the initial survey was sent to a panel of four topic experts for review and assessment of each individual question. The reviewers provided feedback and also rated each question by choosing one of the four options: 1 = poor measure; 2 = below average measure; 3 = above average measure; and $4=$ excellent measure. To be included in the final survey, each question must be scored with a ratio of 0.5 or greater, calculated by the number of achieved points over the maximum (i.e., 16 points). This method of assessment followed the design and content validity described by previous studies. ${ }^{29,32}$

\section{Data Analyses and Statistical Tests}

Descriptive statistics were performed by calculating the means and standard deviations regarding the participants' attitudes and beliefs (Part I), and self-reported level of confidence (Part II). Wilcoxon signed-rank tests were applied to examine whether there was any significant change in the outcome from pretest to posttest. Post-hoc paired t-tests were also performed to compare potential differences between pretest and posttest for Parts I and II of the survey, respectively. The level of significance was set at 0.05 .

\section{RESULTS}

Forty-one out of 42 students completed both the pretest and the posttest, making a $97.6 \%$ response rate. The results showed a positive increase in outcome from the pretest $(5.74 \pm 2.01)$ to the posttest $(6.11 \pm 2.21)$. The change did not reach a significant level $(p=0.25)$. Post-hoc analyses revealed increases in outcomes for both Part I and Part II (see Figure 1). The scores related to attitudes and beliefs increased from $4.13 \pm 0.55$ for the pretest to $4.22 \pm 0.48$ for the posttest $(p=0.44)$. There was also an increase in scores for level of confidence from the pretest to the posttest (i.e., $7.34 \pm 0.96$ to $8.01 \pm 0.79 ; p=0.055$ ). None of these increases demonstrated a significant difference.

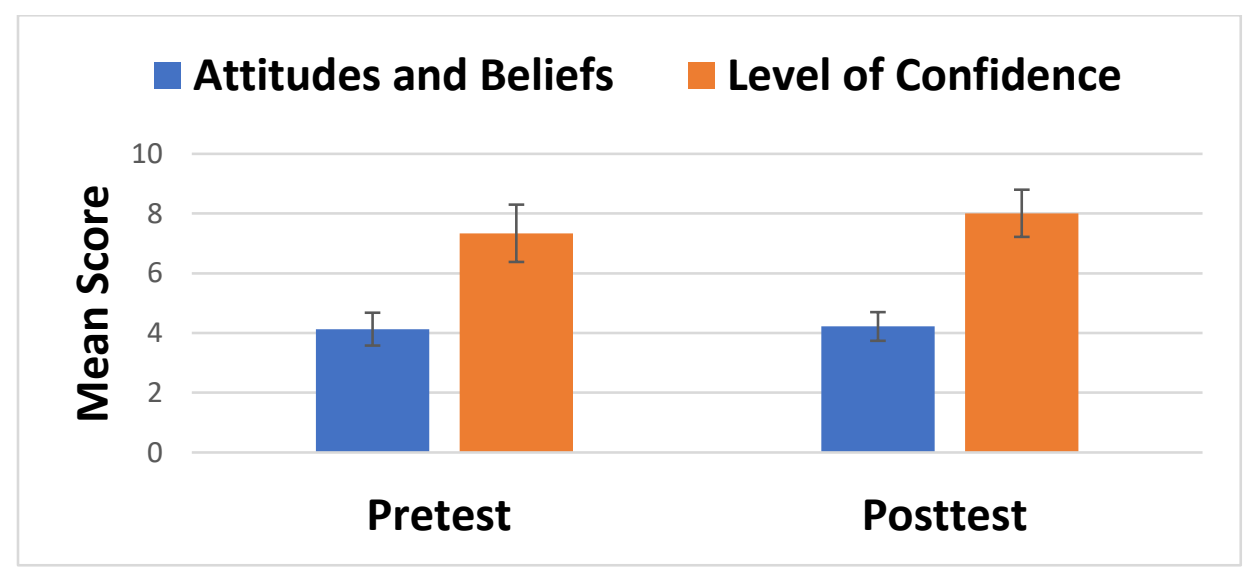

Figure 1. Comparison of students' attitudes and beliefs with self-reported level of confidence in cultural competence between pretest and posttest measures is shown. There were positive increases in both mean scores, but neither reached a statistically significant level $(p=0.44$ and $p=0.055)$. 
There were 61 total responses to two open-ended questions in Part III: 26 responses to Question 1 and 35 responses to Question 2 (see Appendix for questions). Thematic analysis of these responses revealed 1 predominant theme and 2 possible study limitations. The primary theme related to pro bono was a positive benefit from an exposure to other cultures, races, and/or socioeconomic statuses. Two potential limitations that emerged from thematic analysis included a low number of overall exposures and the possibility of diverse prior experiences or previous cultural awareness.

\section{DISCUSSION}

The findings obtained from this study revealed consistent increases in participants' attitudes, beliefs, and level of confidence in cultural competence. Part II of the survey (self-reported level of confidence) showed a greater change compared to that of Part I of the survey (cultural attitudes and beliefs). When compared to previous studies, our study showed similar results to international service-learning and pro bono clinic studies. A majority of these studies showed a positive shift in attitudes, behaviors, and awareness. ${ }^{16-19}$ Other studies completed in a pro bono clinic setting have shown increased development of cultural competence, core values and professionalism. ${ }^{23,24}$ To our knowledge, this is the first study that examined the effect of pro bono clinic experience in first-year physical therapy students. Therefore, the outcome may shed light on the impact of an early intervention such as pro bono clinic experiences through curricular modification.

Although the positive impact of the pro bono clinic experience did not yield a statistically significant effect in this study, clinical significance was evident and meaningful. The benefit was perhaps most apparent in the comments of Part III of the survey results. One participant commented, "I think this experience definitely provided me with an increased understanding of individuals with a different culture/race/social background," while another participant highlighted the experiences' direct impact on cultural competence, "I think this experience improved my cultural competency because I got to work with individuals who are different than myself and different than other individuals that I would otherwise usually prefer or typically work with." This point was further illustrated by another response, "This experience improved my cultural competency by forcing me to recognize and then suppress behaviors in myself of stereotyping patient populations." These comments have captured how the pro bono clinic experience may impact student's understanding of cultural competence by exposing preferential bias and stereotyping of patients.

The outcomes of this study potentially provide useful information to guide the design of culturally competent curriculum across nursing and allied health education programs. This study lends further support for growing evidence about the positive influence of pro bono clinic experiences on cultural competence. Pro bono clinic experience reported in this study involved up to three attendances that offered fairly limited exposure and experience to study participants, yet there were observed increases in the outcomes. One participant commented, "While there is diversity at [the clinic], I don't believe we spend enough time there to really achieve an increased understanding of other cultures/races/social backgrounds." Another response indicated that "The experience might have increased my cultural competency more if we had more visits to the clinic." It may be plausible to suggest that increased experience and visits in the clinic may lead to significant and impactful changes in outcome measures.

This study provides new insights into the attitudes, beliefs, and confidence of first-year health professional students' self-perceived cultural competence. Although the study results were not statistically significant, we believe that experiences in a pro bono clinic early in curriculum can have beneficial effects. We suggest that future studies look to refine the most effective dosage of pro bono clinic participation on the development of cultural competence (e.g., 6 clinic visits instead of 3, or over an entire school year as opposed to just one semester). Future research design can be made more robust by engaging a larger and more geographically and ethnically diverse sample from several institutions. Likewise, a thematic pre-brief or debrief around the topic of cultural competence may prime participants for a more impactful experience. Some of the narrative responses in this study were suggestive of this point and led us to recommend that future studies use a quantitative and qualitative mixed-methods design to further explore these insights. Information collected from the qualitative approach may provide a more thorough understanding and valuable insiders' views than allowed by a quantitative approach alone. Lastly, we encourage formal learning assessment within pro bono clinics, especially as it relates to cultural competence.

\section{Limitations}

There were notable limitations to this study. First, no control group was incorporated. Second, the study was performed over several months during which many life events unrelated to the pro bono clinic could affect cultural competence, potentially confounding the study results. Further, patient cancellations caused even less exposure to some participants. In addition, there was no standardized amount of care provided during each clinic visit. Volume of care varied as it was largely dependent on complexity of the patient case; more complicated patient management may offer less opportunity for novice student interaction and lend more to observation of care provided by more experienced peers or faculty. 


\section{CONCLUSION}

In conclusion, measuring cultural competence development among health professional students is important due to the rapidly growing needs of interacting with an ethnically and socioeconomically diverse patient population. More studies on effect of pro bono clinic toward cultural competence related to allied health professions are warranted, as it appears a very promising means to teaching cultural competence and engaging students in complex clinical scenarios that contextualize learning and have the potential to accelerate growth compared to other means. Healthcare educators need more evidence obtained from educational research to train future culturally competent clinicians, in order to achieve patient-centered, high-quality care and improved outcomes.

\section{REFERENCES}

1. Quick Facts United States. Accessed March 4, 2021. https://www.census.gov/quickfacts/fact/table/US/RHI825218\#RHI825218

2. Cultural respect. Accessed March 4, 2021. https://www.nih.gov/institutes-nih/nih-office-director/office-communicationspublic-liaison/clear-communication/cultural-respect

3. Bernheim SM, Ross JS, Krumholz HM BE. Influence of patients' socioeconomic status on clinical management decisions: a qualitative study. Ann Fam Med. 2008;6:53-59.

4. Brach C, Fraser I. Reducing disparities through culturally competent health care: an analysis of the business case. Qual Manag Heal Care. 2002;10(4):15-28. doi:10.1016/j.physbeh.2017.03.040

5. Betancourt J, Corbett J, Bondaryk M. Addressing disparities and achieving equity: cultural competence, ethics, and health-care transformation. Chest. 2014;145(1):143-148.

6. Bellamy J. Achieving cultural competence. Accessed February 22, 2021. https://www.apta.org/patient-care/publichealth-population-care/cultural-competence/achieve-cultural-competence

7. Cultural competence in physical therapy. Accessed March 4, 2021. https://www.apta.org/patient-care/public-healthpopulation-care/cultural-competence

8. CAPTE Standards and Elements. Accessed February 18, 2021. http://www.capteonline.org/uploadedFiles/CAPTEorg/About_CAPTE/Resources/Accreditation_Handbook/CAPTE_PTSt andardsEvidence.pdf

9. $\quad$ Purnell L. The Purnell model for cultural competence. J Multicult Nurs Heal. 2005;11(2):7-15.

10. Purnell L. The Purnell model for cultural competence. J Transcult Nurs. 2002;13(3):193-196; discussion 200-1.

11. Sasnett $B$, Royal $P$, Ross $T$. Introduction of a cultural training experience into interdisciplinary health science education program. J Allied Heal. 2010;39(2):e55-60.

12. Black J, Purnell L. Cultural competence for the physical therapy professional. J Phys Ther Educ. 2002;16(1):3-10.

13. Rockich-Winston $\mathrm{N}$, Wyatt $\mathrm{T}$. The case for culturally responsive teaching in pharmacy curricula. Am J Pharm Educ. 2019;83(8):7423.

14. Chipps JA, Simpson B, Brysiewicz P. The effectiveness of cultural-competence training for health professionals in community-based rehabilitation: A systematic review of literature. Worldviews Evidence-Based Nurs. 2008;5(2):85-94. doi:10.1111/j.1741-6787.2008.00117.x

15. Watt K, Abbott $P$, Reath J. Developing cultural competence in general practitioners: an integrative review of the literature. BMC Fam Pract. 2016;17(1):1-11. doi:10.1186/s12875-016-0560-6

16. Dupre A, Goodgold S. Development of physical therapy student cultural competency through international community service. J Cult Divers. 2007;14(3):126-134.

17. Hayward LM, Li L. Promoting and assessing cultural competence, professional identity, and advocacy in doctor of physical therapy (DPT) degree students within a community of practice. J Phys Ther Educ. 2014;28(1):23-36.

18. Sawyer K, Lopopolo R. Perceived impact on physical therapist students of an international pro bono clinical education experience in a developing country. J Phys Ther Educ. 2004;18(2):40-47.

19. Hayward LM, Charrette AL. Integrating cultural competence and core values: an international service-learning model. J Phys Ther Educ. 2012;26(1):78-89.

20. Nixon-Cave K, Meadows J. Developing cultural competence in DPT students: Is there a best practice educational model to facilitate students achieving cultural competence? J Phys Ther Educ. 2014;28(1):37-39.

21. Wise HH, Yuen K. Effect of Community-based Service Learning on Professionalism in Student Physical Therapists. J Phys Ther Educ. 2013;27(2):58-64.

22. Lattanzi JB, Campbell SL, Dole RL, Palombaro KM. Students mentoring students in a service-learning clinical supervision experience: an educational case report. Phys Ther. 2011;91(10):1513-1524. doi:10.2522/ptj.20100308 
23. Mai JA, Thiele A, O'Dell B, Kruse B, Vaassen M, Priest A. Utilization of an integrated clinical experience in a physical therapist education program. J Phys Ther Educ. 2013;27(2):25-32.

24. Black JD, Palombaro KM, Dole RL. Student experiences in creating and launching a student-led physical therapy pro bono clinic: a qualitative investigation. Phys Ther. 2013;93(5):637-648. doi:10.2522/ptj.20110430

25. Denton JM, Esparza S, Fike DS, Gonzalez J, Denton ML. Improvements in cultural competence through classroom and local cross-cultural service-learning activities. J Phys Ther Educ. 2016;30(2):6-13.

26. Core Values for the Physical Therapist and Physical Therapist Assistant. Accessed March 4, 2021. https://www.apta.org/siteassets/pdfs/policies/core-values-endorsement.pdf

27. Palombaro KM, Dole RL, Black JD. Curricular integration and measurement of cultural competence development in a group of physical therapy students. J Scholarsh Teach Learn. 2015;15(5):82-96.

28. Gilles J, Bishop M, McGehee W, Lulofs-MacPherson K, Dunleavy K. Impact on clinical performance of required participation in a student-run pro bono clinic. J Phys Ther Educ. 2019;33(3):209-214. doi:10.1097/jte.0000000000000083

29. Artino AR, La Rochelle JS, Dezee KJ, Gehlbach H. Developing questionnaires for educational research: AMEE Guide No. 87. Med Teach. 2014;36(6):463-474. doi:10.3109/0142159X.2014.889814

30. Rew L, Becker H, Cookston J, Khosropour S, Martinez S. Measuring cultural awareness in nursing students. J Nurs Educ. 2003;42(6):249-257.

31. Lin C-J, Lee C-K, Huang M-C. Cultural competence of healthcare providers: a systematic review of assessment instruments. J Nurs Res. 2017;25(3):174-186.

32. Zamanzadeh V, Ghahramanian A, Rassouli M, Abbaszadeh A, Alavi-Majd H, Nikanfar A-R. Design and implementation content validity study: development of an instrument for measuring patient-centered communication. J Caring Sci. 2015;4(2):165-178. doi:10.15171/jcs.2015.017

Survey Questionnaire

\section{Appendix}

Part I: This information assesses your attitudes and beliefs towards various aspects of cultural competence.

Please choose response to the following questions:

Strongly Disagree (SD) [1]

Disagree (D)

Neutral $(\mathrm{N})$

[2]

Agree $(A)$

[3]

Strongly Agree (SA)

1. I do not allow my own personal biases change the way I view others of different cultural backgrounds.
SD
D
$\mathrm{N} \quad \mathrm{A}$
SA
[1]
[2]
[3]
[4]
[5]

2. I feel comfortable interacting with others that have a different race or culture than my own.

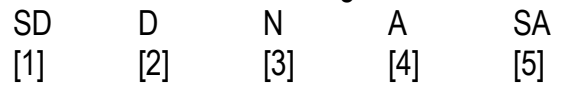

3. I am able to recognize cultural differences between myself and others.

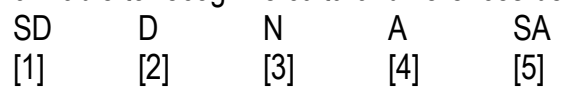

4. I do not get frustrated when interacting with an individual that is culturally different than my own.
$\mathrm{SD}$
D
$\mathrm{N}$
A
SA
[1]
[2]
[3]
[4]
[5]

5. I do not let my personal values to affect the way I view certain cultures.
$\mathrm{SD}$
$\mathrm{N}$
A
$\mathrm{SA}$ 
$[1] \quad[2] \quad[3] \quad[4] \quad[5]$

6. I do not believe that certain races or cultures behave in certain ways or demonstrate stereotypical behaviors.

$\begin{array}{lllll}S D & D & N & A & S A \\ {[1]} & {[2]} & {[3]} & {[4]} & {[5]}\end{array}$

7. I do not participate in insensitive comments or behaviors that disrespect others' cultural beliefs.

$\begin{array}{lllll}S D & D & N & A & S A \\ {[1]} & {[2]} & {[3]} & {[4]} & {[5]}\end{array}$

8. I do not make judgments on a person's character based off of their culture.

$\begin{array}{lllll}S D & D & N & A & S A \\ {[1]} & {[2]} & {[3]} & {[4]} & {[5]}\end{array}$

9. I believe that immigrants can be successful in the U.S. even if they do not speak English.

$\begin{array}{lllll}\text { SD } & D & N & A & S A \\ {[1]} & {[2]} & {[3]} & {[4]} & {[5]}\end{array}$

10. I understand cultural norms can influence communication in different ways such as: eye contact, humor, gestures, silence, etc.

$\begin{array}{lllll}\text { SD } & D & N & A & S A \\ {[1]} & {[2]} & {[3]} & {[4]} & {[5]}\end{array}$

11. I do not jump to conclusions about others before learning more about their culture.

$\begin{array}{lllll}S D & D & N & A & S A \\ {[1]} & {[2]} & {[3]} & {[4]} & {[5]}\end{array}$

12. I let others know that I do not appreciate their comments when they are talking negatively about different cultural groups.

$\begin{array}{lllll}\mathrm{SD} & \mathrm{D} & \mathrm{N} & \mathrm{A} & \mathrm{SA} \\ {[1]} & {[2]} & {[3]} & {[4]} & {[5]}\end{array}$

13. I believe that socioeconomic class and underserved populations may have different motives and values than myself.

$\begin{array}{lllll}S D & D & N & A & S A \\ {[1]} & {[2]} & {[3]} & {[4]} & {[5]}\end{array}$

Part II: Please rate your level of confidence or current experience level in performing the following tasks by selecting the appropriate number, with " 0 " indicating no confidence or experience and " 10 " indicating full confidence or experience.

1. What is your level of confidence when interacting with other people who do not share the same culture or race as yourself?

$\begin{array}{lllllllllll}0 & 1 & 2 & 3 & 4 & 5 & 6 & 7 & 8 & 9 & 10\end{array}$

2. What is your level of confidence to recognize inappropriate comments or actions towards other cultures and/or races?
12
$3 \quad 4$
$\begin{array}{lllll}6 & 7 & 8 & 9 & 10\end{array}$

3. What is your level of confidence when interacting with someone with a different religion than yourself?
1
3
6
7
8
$9 \quad 10$

4. What is your level of confidence with conversing with people who speak different languages?
0
1
2
3
45
6
$\begin{array}{ll}7 & 8\end{array}$
$9 \quad 10$ 
5. What is your level of confidence explaining your own culture to someone from another culture?

$$
\begin{array}{lllllllllll}
0 & 1 & 2 & 3 & 4 & 5 & 6 & 7 & 8 & 9 & 10
\end{array}
$$

6. What is your level of confidence discerning an individual's personality from their cultural stereotypes?

$\begin{array}{lllllllllll}0 & 1 & 2 & 3 & 4 & 5 & 6 & 7 & 8 & 9 & 10\end{array}$

7. What is your level of confidence interacting with populations that are in a lower socioeconomic class than yourself?
0
123
$4 \quad 5$
6
$\begin{array}{lll}7 & 8 & 9\end{array}$

10

8. What is your level of confidence in recognizing disparities in underserved and underrepresented populations?

$\begin{array}{lllllllllll}0 & 1 & 2 & 3 & 4 & 5 & 6 & 7 & 8 & 9 & 10\end{array}$

9. What is your level of confidence interacting with others who may not respect your own culture, beliefs or values?
0
1
23
4
56
$\begin{array}{lll}7 & 8 & 9\end{array}$
10

10. In your opinion, rank your current level of cultural competency.

$\begin{array}{lllllllllll}0 & 1 & 2 & 3 & 4 & 5 & 6 & 7 & 8 & 9 & 10\end{array}$

\section{Post Test Only}

Part III: This narrative portion of the survey aims to get a better understanding of your personal experience with participating in the clinic.

1. In the space below, express your experience in the clinic and touch on whether you thought this experience gave you a better understanding of working with a different culture/race/social group.

2. Describe how this experience may or may not have improved your cultural competence. 